\title{
Similarity solutions for radiation in time-dependent relativistic flows
}

\author{
L. B. Lucy
}

\author{
Astrophysics Group, Blackett Laboratory, Imperial College London, Prince Consort Road, London SW7 2AZ, UK \\ e-mail: 1.lucy@imperial.ac.uk
}

Received 24 August 2004 / Accepted 31 August 2004

\begin{abstract}
Exact analytic solutions are derived for radiation in time-dependent relativistic flows. The flows are sphericallysymmetric homologous explosions or implosions of matter with a grey extinction coefficient. The solutions are suitable for testing numerical transfer codes, and this is illustrated for a fully relativistic Monte Carlo code.
\end{abstract}

Key words. radiative transfer - methods: analytical - methods: numerical

\section{Introduction}

In a recent paper (Lucy 2005), a Monte Carlo (MC) treatment accurate to $O(v / c)$ of the time-dependent transport of radiation in 3-D SNe is described and tested. A major concern in that paper is establishing the accuracy of the MC code. To this end, the 3-D code was applied to a 1-D problem that could be solved independently with conventional numerical methods. Specifically, the test problem was to compute the bolometric light curve of a spherical SN in which the transfer of UVOIR radiation is treated with a grey extinction coefficient. An independent approach to this problem is provided by Castor's (1972) co-moving frame (cmf) moment equations for spherically-symmetric flows. The resulting pair of partial differential equations (PDEs) were solved with the Henyey method.

The final outcome of this test was entirely satisfactory: the mean difference between the two light curves is $\lesssim 0.01$ mag. for elapsed times $t$ from 10 to 50 days. Nevertheless, the differences were initially significant, and it was not clear which code was in error. This question was eventually answered by testing each code separately against an exact similarity solution of Castor's equations. The cause of the differences could then be traced to the poor spatial resolution of the MC code.

In the interest of concise presentation, the essential part played by this similarity solution was not described in the earlier paper (Lucy 2005). But subsequently, this solution was found to generalize to all orders of $v / c$. Accordingly, since time-dependent relativistic flows and the associated transfer problems are of interest for such phenomena as gamma-ray bursts and micro-quasars, this paper derives this solution (and variants thereof) and illustrates its use in testing a relativistic transfer code.

\section{Basic equations}

Similarity solutions will be sought for the frequency-integrated radiation field in a homologously expanding or contracting flow. The configuration is spherically-symmetric, and the matter has grey extinction $(\chi)$ and integrated emissivity $(\eta)$ coefficients that are isotropic in the $\mathrm{cmf}$.

\subsection{The transfer equation}

Mihalas (1980) has derived the general time-dependent $\mathrm{cmf}$ transfer equation for spherically-symmetric flows with relativistic velocities. From this, he derives the zeroth and first frequency-integrated moment equations, whose earlier derivation by Prokof'ev (1962) is acknowledged. When terms of $O\left(v^{2} / c^{2}\right)$ and higher are neglected, Castor's (1972) moment equations are recovered.

Following Mihalas (1980), the interaction coefficients $(\chi, \eta)$ and the radiation field $(I, J, H, K)$ are expressed in the $\mathrm{cmf}$ but spacetime coordinates $(r, t)$ and flow velocities $(v)$ are measured in the rest frame (rf). Since radiation quantities always refer to the cmf, primes or suffixes to indicate this frame are omitted (cf. Mihalas 1980, Sect. III).

In a homologous spherical flow, we have $v=r / t$. For $t>0$, the flow is an explosion $(v>0)$ starting with infinite density at $t=0$. For $t<0$, the flow is an implosion $(v<0)$ leading to infinite density at $t=0$.

When Eq. (2.12) of Mihalas (1980) is applied to the homologous flow of matter with a grey extinction coefficient, the resulting frequency-integrated transfer equation is

$\frac{\gamma}{c}(1+\beta \mu) \frac{\mathrm{d} I}{\mathrm{~d} t}+\frac{\mu}{\gamma c t} \frac{\partial I}{\partial \beta}+\frac{\gamma}{\beta c t}\left(1-\mu^{2}\right) \frac{\partial I}{\partial \mu}+\frac{4 \gamma}{c t} I=\eta-\chi I$ 
Here the dependent variable is the integrated specific intensity $I(\mu ; \beta, t)$, the radial coordinate $r$ has been replaced by $\beta=v / c$, the time derivative is Lagrangian, and $\gamma=1 / \sqrt{1-\beta^{2}}$.

\subsection{Moment equations}

Moment equations can be derived from Eq. (1) as usual by multiplying by $\frac{1}{2} \mu^{m}$ with $m=0,1,2, \ldots$ and then integrating over direction cosine $\mu$. Alternatively, Eqs. (2.16) and (2.17) in Mihalas (1980) can be simplified to the case of homologous flow, as above for his transfer equation.

The resulting equations are

$$
\frac{\gamma}{c} \frac{\mathrm{d} J}{\mathrm{~d} t}+\frac{\gamma \beta}{c} \frac{\mathrm{d} H}{\mathrm{~d} t}+\frac{1}{\gamma c t} \frac{\partial H}{\partial \beta}+\frac{2 \gamma}{\beta c t}(H+2 \beta J)=\eta-\chi J
$$

for the zeroth moment, and

$$
\frac{\gamma}{c} \frac{\mathrm{d} H}{\mathrm{~d} t}+\frac{\gamma \beta}{c} \frac{\mathrm{d} K}{\mathrm{~d} t}+\frac{1}{\gamma c t} \frac{\partial K}{\partial \beta}+\frac{\gamma}{\beta c t}(3 K-J)=-\chi H
$$

for the first moment.

Following Mihalas (1980), Eddington's flux variable $H$ is here preferred to the standard flux $F=4 H$. The integrated physical flux in the cmf is therefore $\mathcal{F}=4 \pi H$.

Comparing Eqs. (2) and (3) with Eqs. (2.16) and (2.17) of Mihalas (1980), we see that specializing to homologous flow has resulted in a huge simplification in the coefficients of the moments $J, H$ and $K$. Most notably, the coefficient of $K$ in the zeroth moment equation reduces to zero, so now only $J$ and $H$ appear in this equation.

\subsection{Separation of variables}

In order to construct simple solutions of these equations for use in testing computer codes, we first effect a separation of variables. This is achieved by writing the specific intensity

$I(\mu ; \beta, t)=I_{1}(\mu ; \beta)\left(t_{1} / t\right)^{p}$

where $t_{1}$ is an arbitrary reference time, and the exponent $p$ is unspecified. With this assumption, each term on the left-hand side of Eq. (1) scales as $\left(t_{1} / t\right)^{p+1}$. Accordingly, we must assume that

$$
\eta(\beta, t)=\eta_{1}(\beta)\left(t_{1} / t\right)^{p+1}
$$

and

$\chi(\beta, t)=\chi_{1}(\beta)\left(t_{1} / t\right)$.

Not surprisingly, this necessary scaling of the extinction coefficient per unit volume implies that the ratio of $1 / \chi$, the local mean free path of a photon, to the radius of the configuration is time-independent.
When Eqs. (4)-(6) are substituted in Eq. (1), the scaling factor cancels by construction, and we thus obtain the transfer equation

$$
\begin{array}{r}
\frac{\mu}{\gamma c t_{1}} \frac{\partial I_{1}}{\partial \beta}+\frac{\gamma}{\beta c t_{1}}\left(1-\mu^{2}\right) \frac{\partial I_{1}}{\partial \mu}+\frac{\gamma}{c t_{1}}[4-p(1+\beta \mu)] I_{1} \\
=\eta_{1}-\chi_{1} I_{1}
\end{array}
$$

that determines the scale-free radiation field $I_{1}(\mu ; \beta)$.

The moments $J, H$ and $K$ evidently also scale as $\left(t_{1} / t\right)^{p}$. The equations satisfied by the corresponding scale-free moments $J_{1}(\beta), H_{1}(\beta)$ and $K_{1}(\beta)$ are

$$
\begin{array}{r}
\frac{1}{\gamma c t_{1}} \frac{\mathrm{d} H_{1}}{\mathrm{~d} \beta}+\frac{\gamma}{\beta c t_{1}}\left(2-p \beta^{2}\right) H_{1}+\frac{\gamma}{c t_{1}}(4-p) J_{1} \\
=\eta_{1}-\chi_{1} J_{1}
\end{array}
$$

for the zeroth moment, and

$$
\begin{aligned}
\frac{1}{\gamma c t_{1}} \frac{\mathrm{d} K_{1}}{\mathrm{~d} \beta}-\frac{\gamma \beta}{c t_{1}} p K_{1}+\frac{\gamma}{\beta c t_{1}}\left(3 K_{1}-J_{1}\right)+\frac{\gamma}{c t_{1}} & (4-p) H_{1} \\
= & -\chi_{1} H_{1}
\end{aligned}
$$

for the first moment.

Equations (7)-(9) are the basic equations of this investigation.

\section{Testing a Monte Carlo code}

If $\mathrm{MC}$ techniques are used directly to simulate the physics of radiation transport, then the MC quanta are photons and convergence to the solution of the Radiative Transfer Equation (RTE) requires that $\mathcal{N} \rightarrow \infty$, where $\mathcal{N}$ is the number of photons whose interaction histories are followed. In such a code, in addition to crossings of boundaries, MC quanta are created spontaneously within the computational domain $D$ by sampling the thermal emissivity and may subsequently be destroyed within $D$ by absorption. Because the RTE is directly simulated, testing such MC codes is not fundamentally different from testing a conventional numerical solution of the RTE.

However, for tranfer problems involving interactions between radiation and the internal energy states of matter, there are advantages in taking the MC quanta to be indestructible and indivisible energy ( $\mathcal{E}$-) packets (Lucy 2003, and references therein). In such a code, in addition to crossings of boundaries, $\mathcal{E}$-packets are created spontaneously within $D$ by sampling the net emissivity (i.e., emission minus absorption) but then, though the nature of the contained energy may change, they are not subsequently destroyed within $D$ by absorption.

\subsection{Moment solution}

Let the integrated net emissivity per unit volume at time $t_{1}$ be $4 \pi \tilde{\eta}_{1}$, where

$\tilde{\eta}_{1}(\beta)=\eta_{1}-\chi_{1} J_{1}$.

This quantity creates radiant energy within the configuration by a physical mechanism that need not be specified for test problems. 
If we replace the right-hand side of Eq. (8) by $\tilde{\eta}_{1}$ and eliminate $J_{1}$ from the left-hand side by setting $p=4$, the result is an ordinary differential equation (ODE) for $H_{1}(\beta)$,

$\frac{\mathrm{d} H_{1}}{\mathrm{~d} \beta}+\frac{2 \gamma^{2}}{\beta}\left(1-2 \beta^{2}\right) H_{1}=\gamma c t_{1} \tilde{\eta}_{1}$.

This equation can be solved analytically with the integrating factor $\beta^{2} / \gamma^{2}$. The solution satisfying the boundary condition $H_{1}(0)=0$ is

$H_{1}(\beta)=c t_{1} \frac{\gamma^{2}}{\beta^{2}} \int_{0}^{\beta} \tilde{\eta}_{1}(b) b^{2} \sqrt{1-b^{2}} \mathrm{~d} b$.

Note that this formula is exact. In transfer theory, analytic formulae for moments are typically not exact because they are derived with Eddington's closure approximation $K=J / 3$. Here this is not necessary: $\mathrm{K}$ and $\mathrm{J}$ drop out of the zeroth moment equation because of the assumptions of homologous flow and scaling exponent $p=4$.

A second point to note is that $H_{1}(\beta)$ has been derived without specifying the scale-free extinction coefficient. Accordingly, Eq. (12) is valid for arbitrary $\chi_{1}(\beta)$.

It is of interest to note that the scaling $p=4$ arises naturally in the limiting case of completely opaque matter within which there is no net emissivity. In this case, the right-hand side of Eq. (2) is zero, and the cmf flux $H=0$ since radiation is position-coupled to matter. The solution of Eq. (2) is then such that $J \propto t^{-4}$, corresponding to adiabatic evolution of the radiation energy density present initially. In contrast, in the solution derived here, this same scaling is maintained because every layer's losses due to flux divergence is exactly replaced by the net emissivity in that layer. Moreover, the similarity solution represents the state reached when initial conditions have been erased.

\subsection{A particular case}

The simplest case for testing a MC code is when $\tilde{\eta}_{1}(\beta)$ is independent of $\beta$. This also simplifies the evaluation of the exact $H_{1}(\beta)$ since the integral in Eq. (12) is then analytic. The result is

$H_{1}(\beta)=\frac{1}{8} c t_{1} \tilde{\eta}_{1} \frac{\gamma^{2}}{\beta^{2}}\left[\sin ^{-1} \beta-\frac{\beta}{\gamma}\left(1-2 \beta^{2}\right)\right]$.

Thus we obtain an exact closed-form expression for the cmf flux in a particular time-dependent relativistic flow.

The corresponding moment $J_{1}(\beta)$ cannot be obtained exactly. But an approximate formula can be derived from Eqs. (9) and (13) with the help of Eddington's closure approximation and surface boundary condition. The resulting formula for $J_{1}$ can then be substituted in Eq. (10) to obtain an approximate formula for the conventional emissivity $\eta_{1}$. Details are omitted.

\subsection{Monte Carlo calculation}

In order to illustrate how similarity solutions can be used to test codes, we now briefly report MC calculations for relativistic homologous flows. The MC code is a spherically-symmetric and fully relativistic version of the 3-D code described recently
(Lucy 2005). As in that code, the MC quanta are indestructible $\mathcal{E}$-packets. The calculations start at reference time $t_{1}$ with no $\mathcal{E}$-packets present. But as time advances, $\mathcal{E}$-packets spontaneously appear in accordance with the net emissivity $\tilde{\eta}_{1}\left(t_{1} / t\right)^{5}$ and then propagate through the configuration interacting with matter in accordance with extinction coefficient $\chi_{1}\left(t_{1} / t\right)$.

We choose to create equal numbers of $\mathcal{E}$-packets in equal intervals of $\log t$ and having $\mathrm{cmf}$ energies $\epsilon(t)$ that are independent of $\beta$. The energy $\mathrm{d} E$ created in the cmf within the space-time element $\mathrm{d} V \mathrm{~d} t$ is $\mathrm{d} E=4 \pi \tilde{\eta} \mathrm{d} V \mathrm{~d} t$. But since $\mathrm{d} V \mathrm{~d} t$ is invariant under the Lorentz transformation, we may regard $\mathrm{d} V \mathrm{~d} t$ as referring to the rf. Accordingly, for the particular case (Sect. 3.2) where $\tilde{\eta}$ does not depend on $\beta$, the total cmf energy created in the rf interval $\mathrm{d} t$ is $4 \pi \tilde{\eta} V \mathrm{~d} t$, where $V(t)=V\left(t_{1}\right)\left(t / t_{1}\right)^{3}$ is the rf volume at time $t$. Thus, if $\mathrm{d} \mathcal{N} / \mathrm{d} \ell n t$ gives the packet creation rate, and we set $p=4$, then the packets' cmf energies are

$\epsilon(t)=4 \pi \eta_{1} t_{1} V_{1}\left(\frac{t_{1}}{t}\right)\left(\frac{\mathrm{d} \mathcal{N}}{\mathrm{d} \ell n t}\right)^{-1}$.

With $\tilde{\eta}$ independent of $\beta, \mathrm{d} E \propto \mathrm{d} V$ at fixed rf $t$. Accordingly, since we choose $\epsilon$ to be independent of $\beta$, equal numbers of packets are created in equal rf volume elements. The appropriate MC sampling algorithm for the initial $\mathrm{rf}$ radius of a packet created at $t$ is $r=R_{*} \sqrt[3]{z}$, where $R_{*}=c \beta_{*} t$ and $z$ is a random number from $(0,1)$. Here $\beta_{*}$ is the value of $v / c$ at the surface.

The transport of these packets through the configuration is as described in the earlier paper (Lucy 2005) except that now the factor $\gamma$ in the Doppler formula is restored to make the code fully relativistic. The cmf energies of the packets that escape from the surface in each rf time step $\Delta t$ are summed and then divided by $\Delta t / \gamma_{*}$ to obtain an estimate of the $\mathrm{cmf}$ luminosity $L(t)$

Monte Carlo simulations for two strongly relativistic explosions are plotted in Fig. 1. For these simulations, the rf time steps are $\Delta \log t=0.02$, during each of which $\mathcal{N}=1000$ additional $\mathcal{E}$-packets are created with $\mathrm{cmf}$ energies given by Eq. (14).

The evolution of the cmf luminosity is shown starting at $t_{1}$, together with the predicted result $L=4 \pi R_{*}^{2} \times 4 \pi H_{1}\left(\beta_{*}\right) \times\left(t_{1} / t\right)^{4}$, with $H_{1}\left(\beta_{*}\right)$ from Eq. (13). One solution is the free streaming limit $\left(\chi_{1}=0\right)$ and the second $\left(\chi_{1}=3\right)$ has photon mean free paths $=R_{*}(t) / 3$. In both cases, the MC solutions tend asymptotically to the similarity solution, the convergence being somewhat slower for $\chi_{1}=3$ because of the longer residence times of the $\mathcal{E}$-packets. Since agreement is achieved in both cases, the prediction, for $p=4$, that the $\mathrm{cmf}$ flux $H$ is independent of $\chi_{1}$ is confirmed.

\subsection{Relativistic implosions}

Although relatively trivial, it is of interest to illustrate the application to implosions. Accordingly, Fig. 2 shows the same two test problems but with the signs of $t_{1}$ and $\beta *$ reversed. Again, after a transition period during which the internal radiation field is established, the MC solutions converge to the similarity solution.

This possibility of testing numerical treatments of radiative transfer in relativistic implosions is possibly relevant for codes 


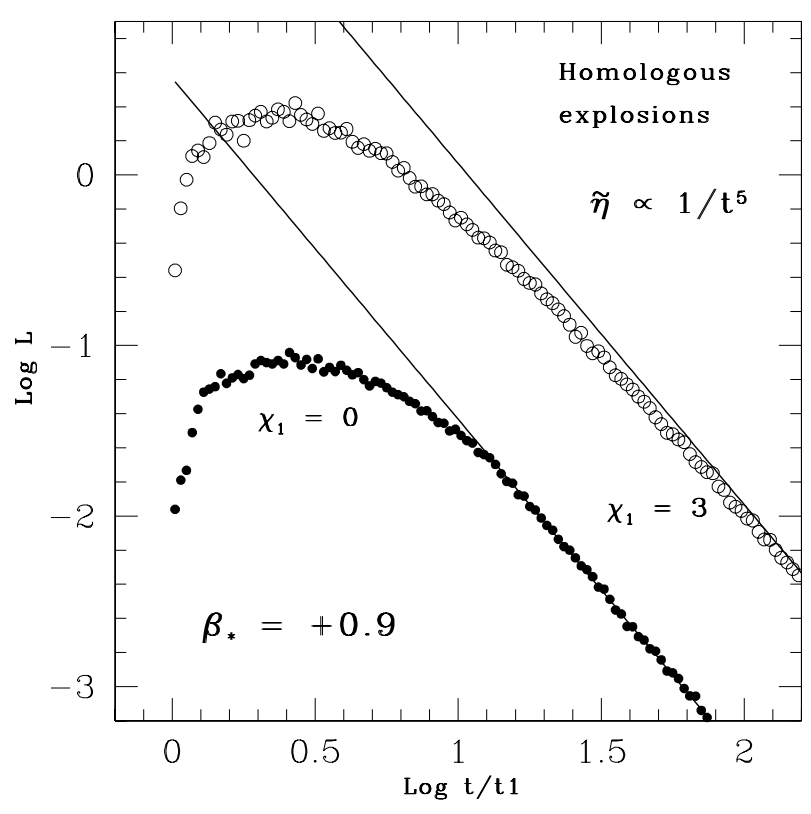

Fig. 1. Explosions. Comparison of MC calulations (circles) of the cmf luminosity $L(t)$ with predictions of similarity theory (straight lines). The surface velocity $v_{*}=0.9 c$. The unit for the indicated scalefree extinction coefficients $\chi_{1}$ is $1 / R_{1}$, where $R_{1}=v_{*} t_{1}$. The unit of luminosity is $4 / 3 \pi R_{1}^{3} \times 4 \pi \tilde{\eta}_{1}$. The luminosites for $\chi_{1}=3$ have been increased by 1.5 dex.

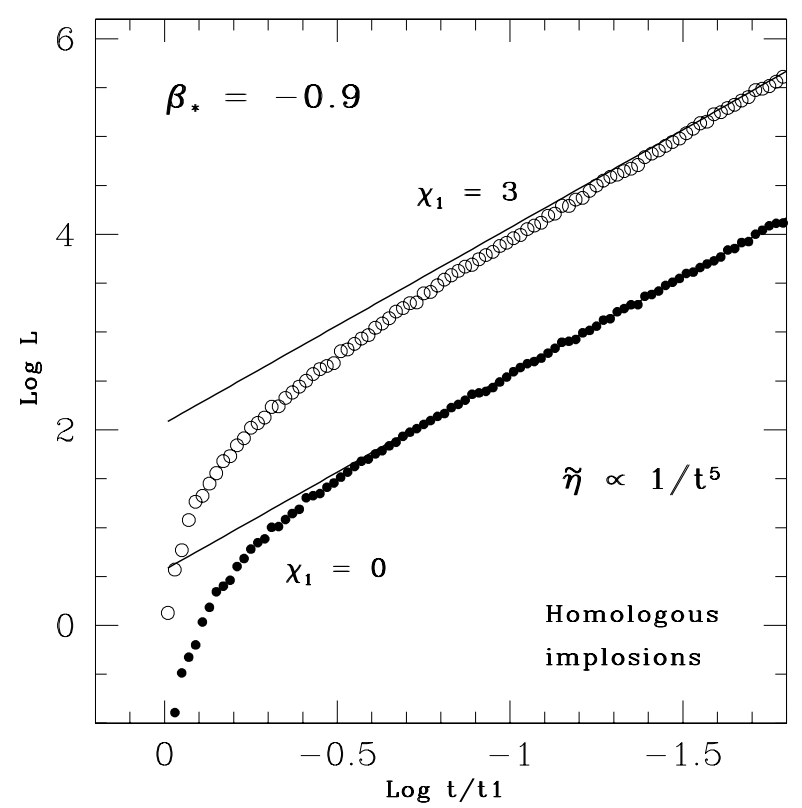

Fig. 2. Implosions. Same as Fig. 1 but with signs reversed for $\beta_{*}$ and $t_{1}$. The luminosites for $\chi_{1}=3$ have been increased by $1.5 \mathrm{dex}$.

that simulate laser-driven implosions in support of the quest for fusion by inertial confinement.

Accurate numerical solutions for relativistic inflows have also been computed by Yin \& Miller (1995), who stress the dramatic effects that can arise from photon trapping. But their solutions are only for stationary flows.

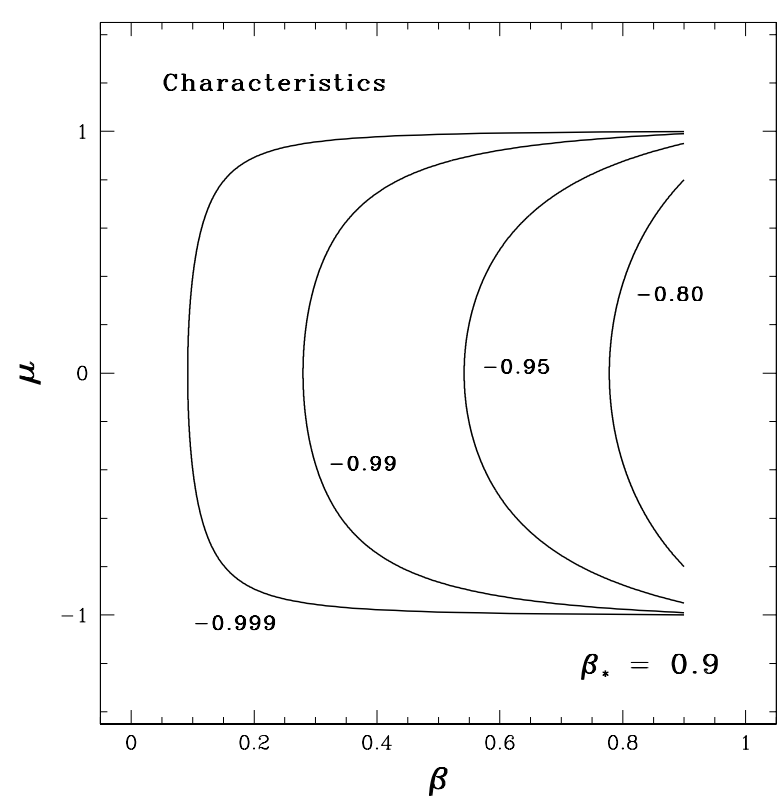

Fig. 3. Characteristic trajectories $\mu\left(\beta ; \mu_{*}\right)$ for Eq. (7) when $\beta_{*}=0.9$. The curves are labelled with the value of $\mu_{*}$, the direction cosine at the surface for the inward ray.

\section{Testing a transfer code}

Apart from the degenerate case $\chi_{1}=0$, the exact solution of Sect. 3 is not appropriate for the precision testing of a conventional relativistic transfer code since the implied scalefree emissivity $\eta_{1}$ could only be determined approximately. Accordingly, we now seek exact solutions when $\eta_{1}$ is specified rather than $\tilde{\eta}_{1}$.

\subsection{Solution along characteristics}

As did Mihalas (1980, Sect. IIIb) for his general equation, we construct characteristics for Eq. (7) such that the partial differential operator becomes a perfect differential. Thus Eq. (7) becomes

$\frac{1}{c t_{1}} \frac{\mathrm{d} I_{1}}{\mathrm{~d} s}+\left[\chi_{1}-\frac{(p-4) \gamma+p \gamma \beta \mu}{c t_{1}}\right] I_{1}=\eta_{1}$

where $c t_{1} s$ is distance along a characteristc defined by the equations

$\frac{\mathrm{d} \beta}{\mathrm{d} s}=\frac{\mu}{\gamma} \quad$ and $\quad \frac{\mathrm{d} \mu}{\mathrm{d} s}=\frac{\gamma}{\beta}\left(1-\mu^{2}\right)$.

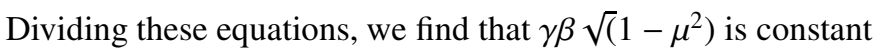
along characteristics. Accordingly, the family of characteristics is given by

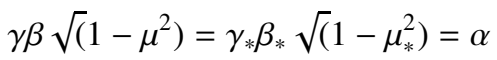

where $-1<\mu_{*}<0$ is a characteristic's direction cosine at its entry point into the configuration. Integrations of Eq. (15) proceed inwardly along such characteritics starting at $s=0$ with the boundary condition $I_{1}\left(\mu_{*} ; \beta_{*}\right)=0$ and continuing until the characteristic emerges at the surface with $\mu=\left|\mu_{*}\right|$.

In Fig. 3, the characteristics are plotted for various values of $\mu_{*}$ when $\beta_{*}=0.9$. Note that, to obtain a characteristic that 


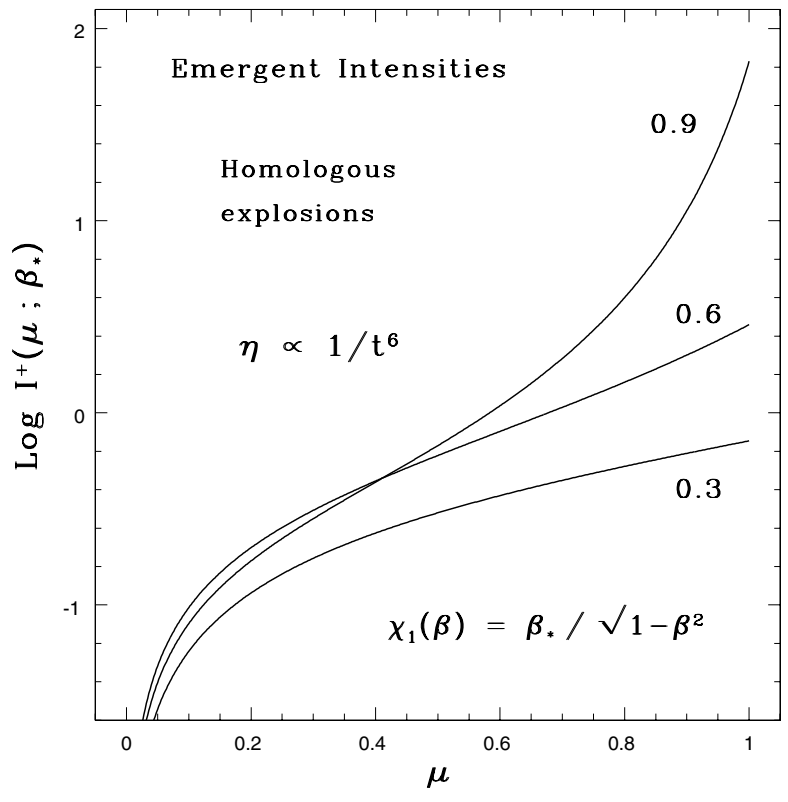

Fig. 4. Emergent intensities in the co-moving frame for the indicated values of $\beta_{*}$. The exponent $p=5$, and $\chi_{1}$ is from Eq. (24). The formula for $\chi_{1}(\beta)$ in units of $1 / R_{1}$ is exhibited (cf. Figs. 1 and 2). The unit of intensity is $c t_{1} \eta_{1}$.

penetrates close to the centre, the parameter $\mu_{*}$ must closely approach -1 . The point of closest approach $(\mu=0)$ is at $\gamma=$ $\sqrt{1+\alpha^{2}}=\gamma_{0}$, or, equivalently, at $\beta=\alpha / \gamma_{0}=\beta_{0}$.

Parenthetically, we note that these analytic characteristic curves provide a further powerful test of the relativistic MC code described in Sect. 3.3. In the free streaming case, each $\mathcal{E}$-packet follows its appropriate characteristic to high precision, as it should. At creation, an $\mathcal{E}$-packet's initial $\beta$ and $\mu$ determine its invariant $\gamma \beta \sqrt{\left(1-\mu^{2}\right)}$ and hence $\mu_{*}$ from Eq. (17). The packet then propagates along this characteristic in the direction of increasing $s$ until it escapes at the surface $\beta=\beta_{*}$ with $\mu=\left|\mu_{*}\right|$.

The simplicity of the characteristics for homologous flow allows the dimensionless arc length $s$ to be evaluated analytically as a function of $\beta$. Integrating the first member of Eq. (16) after eliminating $\mu$ with Eq. (17), we find that, along the inwardly-directed $(\mu<0)$ segment of a characteristic,

$s\left(\beta ; \mu_{*}\right)=\frac{1}{\gamma_{0}}\left[\sin ^{-1}\left(\frac{\gamma_{0}}{\gamma}\right)-\sin ^{-1}\left(\frac{\gamma_{0}}{\gamma_{*}}\right)\right]$.

The corresponding formula after the point of closest approach $(\mu>0)$ is

$s\left(\beta ; \mu_{*}\right)=\frac{1}{\gamma_{0}}\left[\pi-\sin ^{-1}\left(\frac{\gamma_{0}}{\gamma}\right)-\sin ^{-1}\left(\frac{\gamma_{0}}{\gamma_{*}}\right)\right]$.

Because the function $s\left(\beta ; \mu_{*}\right)$ is readily inverted to give $\beta\left(s ; \mu_{*}\right)$, the remaining quantities $\gamma, \mu, \eta_{1}(\beta)$ and $\chi_{1}(\beta)$ can likewise be transformed into functions of $s$ along the characteristic defined by $\mu_{*}$ for the given $\beta_{*}$; and this remark therefore applies also to the coefficient of $I_{1}$ in Eq. (15).

Let us now define the effective extinction coefficient along a characteristic to be

$\hat{\chi}_{1}\left(s ; \mu_{*}\right)=\chi_{1}-\frac{(p-4) \gamma+p \gamma \beta \mu}{c t_{1}}$ with corresponding effective optical depth

$\hat{\tau}_{1}\left(s ; \mu_{*}\right)=c t_{1} \int_{0}^{s} \hat{\chi}_{1} \mathrm{~d} s$.

In terms of these quantities, the formal solution of Eq. (15) subject to the boundary condition $I=0$ at $s=0$ is

$I_{1}\left(s ; \mu_{*}\right)=c t_{1} \int_{0}^{s} \eta_{1}\left(s^{\prime}\right) \mathrm{e}^{\hat{\tau}\left(s^{\prime}\right)-\hat{\tau}(s)} \mathrm{d} s^{\prime}$.

With straightforward numerical integrations, Eqs. (18)-(22) allow the intensity $I\left(s ; \mu_{*}\right)$ to be determined as a function $s$ along the characteristic defined by $\mu_{*}$. By varying $\mu_{*}$ from -1 to 0 , we can thus determine $I$ throughout the $(\mu, \beta)$-plane.

This reduction of the problem to solution with the formal integral strongly suggests that these results could also be obtained with the method developed by Baschek et al. (1997). Although their paper is restricted to stationary relativistic flows, they note that time-dependent problems can be treated.

\subsection{Equivalent static medium}

The above analysis shows that, with the scaling assumptions for $\eta$ and $\chi$ given in Eqs. (5) and (6), the time-dependent relativistic transfer problem for homologous spherical flow, reduces asymptotically to a tranfer problem in a static medium. For explosions, the solution tends to this asymptote as $t \rightarrow \infty$; for implosions, the limit is $t \rightarrow 0$. For free-streaming, the similarity solution is achieved after all light signals emitted from within the configuration at $t=t_{1}$ have escaped.

Because light travel-time and relativistic effects are absent in a static medium, these effects reappear in Eq. (20) as corrections to the extinction coefficient. The term $\propto(p-4)$, which survives in the limit $\beta \rightarrow 0$, represents the combination of finite propagation speed and the time-dependence of the emissivity. The second correction term, which $\rightarrow 0$ as $\beta \rightarrow 0$, represents relativistic effects.

Note that these corrections can give $\hat{\chi}_{1}<0$, which implies that $\hat{\tau}_{1}$ is not necessarily a monotonically increasing function of $s$. Accordingly, $\hat{\tau}_{1}$ is not appropriate as an alternative independent variable for Eq. (15).

\subsection{A particular case: Moment solution}

Although the analysis of Sect. 4.1 provides a complete solution for the scale-free radiation field without assumptions about $\eta_{1}(\beta)$ or $\chi_{1}(\beta)$, the answer is not in closed analytic form as was the earlier result (Eq. (13)) for the cmf flux $H_{1}$ when the net emissivity $\tilde{\eta}$ is independent of $\beta$. Interestingly, an analogous result can be constructed when the conventional emissivity $\eta$ is the quantity specified.

The coefficient of $J_{1}$ in Eq. (8) is zero if we set

$\chi_{1}(\beta)=(p-4) \frac{\gamma}{c t_{1}}$.

Thus $p>4$ is necessary for $\chi_{1}>0$, while $p=4$ gives freestreaming radiation. 
With $\chi_{1}$ given by Eq. (23), Eq. (8) simplifies to

$\frac{\mathrm{d} H_{1}}{\mathrm{~d} \beta}+\frac{\gamma^{2}}{\beta}\left(2-p \beta^{2}\right) H_{1}=c t_{1} \gamma \eta_{1}$.

This equation has integrating factor $\beta^{2} / \gamma^{p-2}$, and so the solution satisfying the boundary condition $H_{1}(0)=0$ is

$H_{1}(\beta)=c t_{1} \frac{\gamma^{p-2}}{\beta^{2}} \int_{0}^{\beta} \eta_{1}(b) b^{2}\left(1-b^{2}\right)^{\frac{p-3}{2}} \mathrm{~d} b$.

A simple case for which this integral is analytic is obtained by assuming that $\eta_{1}$ is independent of $\beta$ and that $p=5$. The result is

$H_{1}(\beta)=\frac{1}{15} c t_{1} \eta_{1} \gamma^{3} \beta\left(5-3 \beta^{2}\right)$.

With this exact formula, a code that solves relativistic transfer problems with conventional techniques can be subjected to tests similar to those described in Sect. 3.3 for a MC code based on $\mathcal{E}$-packets.

\subsection{A particular case: Complete solution}

The particular case of Sect. 4.2 is remarkable in that the complete solution can be obtained in closed form. The starting point is Eq. (15) with $\chi_{1}$ from Eq. (23). The independent variable $s$ is conveniently transformed to $\beta$ using the first member of Eq. (16). The resulting transfer equation is

$\frac{\mathrm{d} I_{1}}{\mathrm{~d} \beta}-p \gamma^{2} \beta I_{1}=c t_{1} \eta_{1} \frac{\gamma}{\mu}$

where, from Eq. (17),

$\mu(\beta)= \pm \frac{\gamma_{0}}{\beta} \sqrt{\beta^{2}-\beta_{0}^{2}}$.

Here the negative root applies along the inward segment of a characteristic as $\beta$ decreases from $\beta_{*}$ to $\beta_{0}-$ see Fig. 3 . Thereafter, the positive root applies.

The integrating factor for Eq. (27) is $\gamma^{-p}$. Accordingly, substituting for $\mu$, imposing boundary condition $I^{-}\left(\beta_{*}\right)=0$, setting $p=5$, and assuming that $\eta_{1}$ is independent of $\beta$, we find that the intensity of inwardly-directed radiation is

$I^{-}\left(\beta ; \mu_{*}\right)=c t_{1} \eta_{1} \frac{\gamma^{5}}{\gamma_{0}} \int_{\beta}^{\beta_{*}} \frac{\left(1-b^{2}\right)^{2}}{\sqrt{b^{2}-\beta_{0}^{2}}} b \mathrm{~d} b$

which simplifies to

$I^{-}\left(\beta ; \mu_{*}\right)=c t_{1} \eta_{1} \frac{\gamma^{5}}{\gamma_{0}}\left[g\left(\beta_{*} ; \mu_{*}\right)-g\left(\beta ; \mu_{*}\right)\right]$

where

$g\left(\beta ; \mu_{*}\right)=\xi\left(\frac{1}{\gamma^{4}}+\frac{4}{3} \frac{\xi^{2}}{\gamma^{2}}+\frac{8}{15} \xi^{4}\right)$

with

$\xi\left(\beta ; \mu_{*}\right)=\sqrt{\beta^{2}-\beta_{0}^{2}}$.
The outwardly-directed intensity is obtained similarly. In this case, the boundary condition is $I^{+}\left(\beta_{0} ; \mu_{*}\right)=I^{-}\left(\beta_{0} ; \mu_{*}\right)$, and the solution is

$I^{+}\left(\beta ; \mu_{*}\right)=c t_{1} \eta_{1} \frac{\gamma^{5}}{\gamma_{0}}\left[g\left(\beta_{*} ; \mu_{*}\right)+g\left(\beta ; \mu_{*}\right)\right]$.

Equations (30) and (33) determine the intensity along the characteristic defined by $\mu_{*}$. But these formulae can readily be used to calclulate $I_{1}(\mu ; \beta)$, the intensity as a function of $\mu$ at fixed $\beta$. Given $\mu$ and $\beta$, Eq. (17) determines $\alpha$, which in turn determines $\beta_{0}$, so that $\beta_{0}=\beta_{0}(\mu, \beta)$ and similarly of course for $\gamma_{0}$. Thus the right-hand sides of Eqs. (30) and (33) are now functions of $\mu$ and $\beta$, and the characteristics are no longer relevant.

This exact closed form analytic solution has been checked by substitution back into Eq. (7) using numerical differentation to evaluate the two partial derivatives.

In Fig. 4, the emergent intensity given by Eq. (33) is plotted for various values of $\beta_{*}$. This shows extremely strong forward peaking when $\beta_{*}=0.9$, with huge intensities when $\mu \gtrsim 0.9$. This is basically a light travel-time effect: with $\beta_{*}=0.9$, radiation emerging with $\mu \sim 1$ includes photons emitted shortly after the explosion when, since $j \propto 1 / t^{6}$, the emissivity was much higher than that now in the surface layers.

One consequence of this forward peaking is poor accuracy for Eddington's approximations. Thus, at the surface of the $\beta_{*}=0.9$ solution, $K_{1} / J_{1}=0.821$ and $H_{1} / J_{1}=0.895$, as against Eddington's values of $1 / 3$ and $1 / 2$, repectively.

\subsection{A particular case: Thermal emission}

Thus far the physical mechanisms responsible for extinction and emission have not been specified. But let us now suppose that

$\chi=k+\sigma$

where $k$ and $\sigma$ are grey absorption and scattering coefficients, respectively. The corresponding integrated emissivity coefficient is then

$\eta=k B+\sigma J$

where $B$ is the integrated Planck function, and the scattering is isotropic in the cmf.

Equations (34) and (35) are consistent with the scaling of Eqs. (5) and (6) if $k$ and $\sigma$ are $\propto 1 / t$ and if $B \propto 1 / t^{p}$. Given these scalings, the exact solution of Sect. 4.4 can now be applied as follows: we set $p=5$ and choose the scale-free functions $k_{1}(\beta)$ and $\sigma_{1}(\beta)$ such that their sum satisfies Eq. (23). Then, since $J_{1}(\beta)$ can be computed from Eqs. (30) and (33), the implied scale-free function $B_{1}(\beta)$ can be derived from Eq. (35). Because the thermal emissivity is now known, this analytic solution can be used to test a code that incorporates an iteration procedure to detemine the scattering contribution to the emissivity.

The net emissivity $\tilde{\eta}_{1}=\eta_{1}-\chi_{1} J_{1}=k\left(B_{1}-J_{1}\right)$ is also determined by the above steps. Accordingly, this analytic solution can be used to test MC codes based on $\mathcal{E}$-packets. Moreover, this test is in principle more powerful than that provided by the flux moment solution of Sect. 3.2 since the angular distribution of the MC radiation field can now also be checked. 


\section{Conclusion}

The aim of this paper has been to derive exact analytic solutions in order to test radiative transfer codes for relativistic flows. Such solutions exist for spherically-symmetric homologous flows with power-law time dependencies for the grey extinction coefficient and the integrated emissivity. The exact solution for the integrated intensity derived in Sect. 4.4, being a function of three independent variables $t, \mu, \beta$ and one parameter $\beta_{*}$, provides an extraordinarily demanding and informative test for such codes. Moreover, its somewhat contrived derivation based on a particular spatial variation of $\chi$ in no way lessens its usefulness.

In addition to deriving closed form analytic formulae for the intensity and flux in the cmf, an exact formula has been derived for the characteristics. This allows the kinematic and geometric aspects of relativistic transport codes to be tested independently of the treatments of absorption and emission. For MC codes, this test is carried out by setting the extinction coefficient to zero and then checking that photon packets propagate along the known characteristics.
In solving time-dependent transport problems with the RTE, the time derivatives are commonly approximated with a backward difference formula, thus using the solution at the previous time step. This introduces errors $O(\Delta t)$ that might well accumulate as the integration proceeds. With the availability of an exact time-dependent solution, the magnitude of the accumulated error can be determined. If the error accumulation is unacceptable, a higher order difference formula can be employed that uses the solutions at the two previous time steps (Lucy 2005).

\section{References}

Baschek, B., Efimov, G. V., von Waldenfels, W., \& Wehrse, R. 1997, A\&A, 317, 630

Castor, J. I. 1972, ApJ, 178, 779

Lucy, L. B. 2003, A\&A, 403, 261

Lucy, L. B. 2005, A\&A, 429, 19

Mihalas, D. 1980, ApJ, 237, 574

Prokof'ev, V. A. 1962, Soviet Phys. - Doklady, 6, 861

Yin, W.-W., \& Miller, G. S. 1995, ApJ, 449, 826 